\title{
Analysis of electron trajectories with two-color strong-field ionization
}

\author{
Jost Henkel and Manfred Lein \\ Institut für Theoretische Physik and Centre for Quantum Engineering and Space-Time Research (QUEST), \\ Leibniz Universität Hannover Appelstraße 2, 30167 Hannover, Germany
}

(Received 17 February 2015; published 24 July 2015)

\begin{abstract}
Two-color ionization of atoms with a strong 800-nm fundamental component and a weak 400-nm component with perpendicular polarization gives detailed insight into the ionization dynamics. When the delay between the two colors is varied on a subcycle scale, the slow-electron signal shows an oscillatory structure due to intracycle interference between different ionization times. Using a trajectory-based interference model, we extract the relative strength of the two contributing pathways. Ionization times can be read directly from the delay scan, and the times for the long trajectories agree well with the quantum-orbit model. The fast electrons arise predominantly from long rescattering trajectories.
\end{abstract}

DOI: 10.1103/PhysRevA.92.013422

PACS number(s): 32.80.Rm, 32.80.Qk

\section{INTRODUCTION}

In the physics of atoms and molecules interacting with strong laser pulses, it has become a standard approach to interpret strongly nonperturbative phenomena in terms of electron trajectories: In a strong field an electron is freed from its bound state and is then driven by the force of the oscillatory field [1]. Depending on the exact timing of ionization and on the initial conditions, different kinds of trajectories are possible. Some of these trajectories return to the parent ion, leading to elastic or inelastic scattering or recombination of the electron with its parent ion. The latter leads to the emission of high-energy radiation in a process called high-harmonic generation (HHG) [2,3]. Numerous applications of strong fields are closely connected to the trajectory-based interpretation of the ionization mechanism. For example, interference of trajectories launched within the same quarter cycle of the laser field leads to photoelectron holography $[4,5]$. The control of trajectories via the carrier-envelope phase allows enhancement or suppression of molecular fragmentation by few-cycle pulses [6]. With HHG spectroscopy temporal information about the recombination timing [7] as well as structural [8,9] or dynamical [10-12] properties of the ion can be extracted. These applications underline the need for precise knowledge about the electron trajectories.

Since HHG and double ionization depend strongly on the recollision event $[3,13,14]$, they can be controlled by introducing a second harmonic field with orthogonal polarization that makes some trajectories miss the parent ion due to the incurred lateral offset $[9,15-17]$. By scanning the subcycle delay between the two fields, a variation of the HHG emission intensity was observed [18]. Analyzing the observables with the help of trajectory models allowed the reconstruction of ionization and recollision times. The experiment showed excellent agreement with the quantumorbit (QO) model [19,20] and lesser agreement with the purely classical model. Theoretical analysis using the timedependent Schrödinger equation (TDSE) also confirmed the QO model [21,22]. Despite strong interest in the subject, only few investigations of the accompanying photoelectrons have been reported for two-color ionization in fields with orthogonal polarization [23,24], compared to fields with parallel polarization [25-32].
A key aspect of strong-field ionization is that different time instants of ionization can produce electrons with the same final momentum. The coherence of the process implies interference between these trajectories. Various types of interference can be distinguished. The periodic repetition of ionization in every full period of the laser field leads to peaks in the photoelectron spectrum separated by the laser frequency, a phenomenon known as above-threshold ionization (ATI) [33]. In contrast to these intercycle interferences, ionization pathways starting in two neighboring quarter cycles of the field can lead to intracycle interference [34,35]. The two pathways are fundamentally different as only electrons from the quarter cycle with decreasing field are able to recollide with the parent ion.

In this article, we present a theoretical analysis of ionization in a two-color field consisting of an 800-nm fundamental field and a weak 400-nm second harmonic field polarized perpendicularly to the first. We show that not only ionization times but also the relative strengths of different pathways can be extracted from the photoelectron distributions. This arises from the clear visibility of intracycle interference in the two-color delay scan.

The paper is organized as follows. Parameters for the laser field and computational details are given in Sec. II. Results and discussion are presented in Sec. III, and the summary is provided in Sec. IV.

\section{PARAMETERS AND COMPUTATIONAL DETAILS}

The two-dimensional TDSE (atomic units are used in the equations)

$$
i \partial_{t} \psi(t)=\left[\frac{1}{2}[\mathbf{p}+\mathbf{A}(t, \phi)]^{2}+V(r)\right] \psi(t),
$$

is solved with the electric field $\mathbf{E}(t)=-\dot{\mathbf{A}}(t)$ chosen as

$$
\mathbf{E}(t)=f(t) E_{0}\left[\hat{\mathbf{e}}_{x} \cos (\omega t)+\hat{\mathbf{e}}_{y} \epsilon \cos (2 \omega t+\phi)\right] .
$$

The frequency $\omega=0.05695$ a.u. corresponds to $800-\mathrm{nm}$ wavelength. The small relative strength $\epsilon=0.1$ of the second harmonic field allows us to view it as a perturbation [18,21]. The field strength is $E_{0}=0.107$ a.u., corresponding to an intensity of $4.0 \times 10^{14} \mathrm{~W} / \mathrm{cm}^{2}$ for the component along $x$. The phase $\phi$ has the role of a delay between the two field components and is scanned between 0 and $2 \pi$. The trapezoidal envelope $f(t)$ consists of a constant part of 6 
fundamental periods and a leading and trailing ramp of two periods each. After propagation of the wave function, starting from the ground state, the photoelectron momentum distribution is calculated as in Ref. [36]. We use a softcore potential $V(r)=-1 / \sqrt{\alpha+r^{2}}$ with the parameter $\alpha$ chosen to reproduce the ionization potential of helium $\left(I_{\mathrm{p}}=\right.$ 0.904 a.u. $=24.6 \mathrm{eV}$ ). Additionally, we have performed calculations with a soft-core potential reproducing the ionization potential of krypton $\left(I_{\mathrm{p}}=0.515\right.$ a.u. $\left.=14.0 \mathrm{eV}\right)$ and for helium with a higher intensity $4.5 \times 10^{14} \mathrm{~W} / \mathrm{cm}^{2}$ to verify the consistency of our results.

\section{RESULTS AND DISCUSSION}

Figure 1(a) gives an example of a momentum distribution from two-color irradiation. In Fig. 1(b) we show the delay dependence of the electron yield in three different energy ranges. Slow electrons that undergo the ionization process without gaining additional energy from a recollision with the parent ion have a maximum energy of $2 U_{\mathrm{p}}$ according to the classical model with zero initial velocity, corresponding to the momentum $p_{x}= \pm E_{0} / \omega= \pm A_{0}$. Here, $U_{\mathrm{p}}=E_{0}^{2} /\left(4 \omega^{2}\right)$
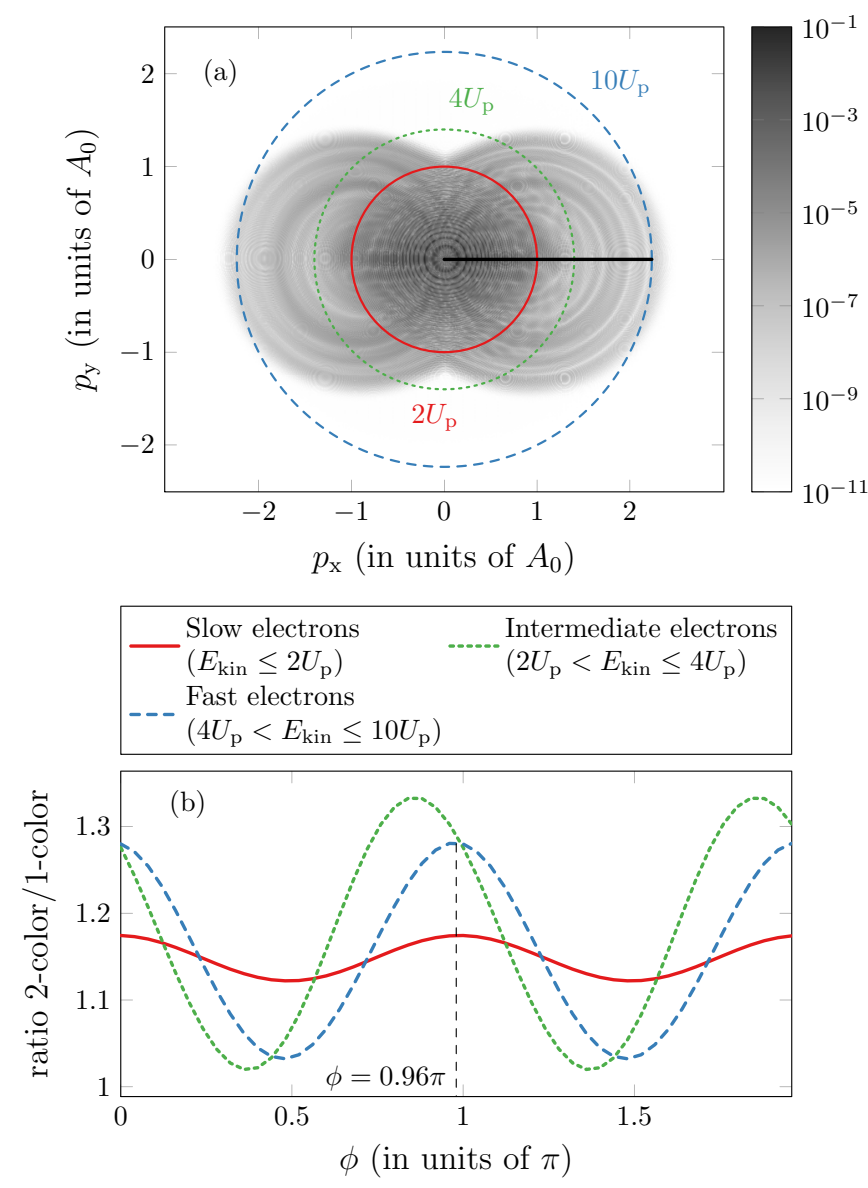

FIG. 1. (Color online) (a) Photoelectron momentum distribution at the two-color delay $\phi=0.96 \pi$, which gives the largest fastelectron yield; the black line shows the positive $p_{x}$ axis and the circles indicate the integration limits used to obtain the results in panel (b). (b) Delay scan of the photoelectron signal integrated over three different energy ranges. The signal is given relative to the signal from an 800-nm single-color field.

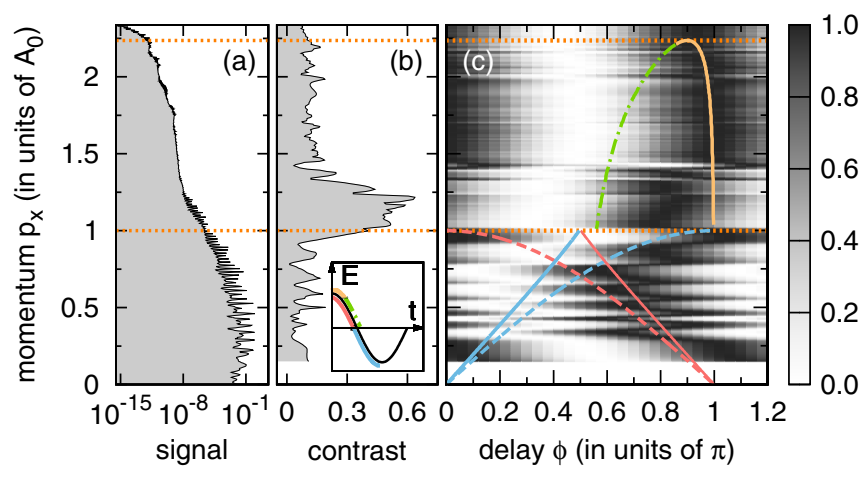

FIG. 2. (Color online) (a) Photoelectron spectrum from the 800-nm single-color field at $p_{y}=0$. (b) Contrast for every momentum of the delay scan. (c) Delay scan at $p_{y}=0$. The two horizontal dotted orange (gray) lines show the classical limits of slow ( $p_{x}=$ $A_{0}=\sqrt{2 U_{\mathrm{p}}}$ ) and fast electrons $\left(p_{x}=\sqrt{2\left(10 U_{\mathrm{p}}\right)}\right.$ [40]). Within the slow electrons two dashed lines (red and blue) give the results for the classical model. The two solid lines give the corresponding solutions of the QO model. The descending branch of the models (red [gray] lines) corresponds to electrons born while the electric field decreases, i.e., electrons able to recollide. At high momenta a classical model for the fast electrons is shown, with the colors indicating the short (dot-dashed green [gray] line) and long trajectories (yellow [light gray] solid line) according to HHG conventions. The inset in panel (b) illustrates the respective ionization times using the same colors.

is the pondermotive potential of the fundamental field. The second harmonic is a weak probing field, which is confirmed by the weak delay dependence of the slow-electron yield. Fast electrons, however, are due to recollisions, and in analogy to the HHG experiments $[15,18]$ the fast-electron yield is strongly delay dependent. The strongest enhancement for electrons above $4 U_{\mathrm{p}}$ is around $\phi \approx 0.96 \pi$ and $\phi \approx 1.96 \pi$, which represents a shift of about $\pi / 2$ compared to HHG [18,21]. We show below that this is due to the dominance of the long rescattering trajectories.

\section{A. Two-color delay scan}

In the following, we restrict our analysis to photoelectrons with zero final lateral momentum $\left(p_{y}=0\right)$. The momentum distribution for single-color ionization is shown in Fig. 2(a). The pulses are sufficiently long to avoid forward-backward asymmetries (effects depending on the carrier-envelope phase of the fundamental field). Therefore, only positive momenta $p_{x}>0$ are analyzed. The calculated two-color delay scan is periodic in $\phi$ with period $\pi$ due to symmetry reasons. For better visual presentation, we have averaged the distribution by integrating over each ATI peak. Figure 2(b) shows the (Michelson) contrast of the $\phi$ dependence for each momentum. In Fig. 2(c), the $\phi$-dependent signal is shown after it has been normalized separately for each momentum $p_{x}$. The signal has been linearly rescaled so that it varies between zero and one. Therefore, Fig. 2(c) has no information on the original modulation depth of the $\phi$ dependence. This information is provided separately by Fig. 2(b).

Slow electrons exhibit rapid oscillations in the delay scan, i.e., a change in the phase of the oscillation with $p_{x}$, whereas the fast electrons show almost no $p_{x}$ dependence. Both energy ranges show a contrast of about 0.1 . At intermediate momenta 
between 0.8 and $1.4 A_{0}$ we have an overlap region where slow and fast electrons have comparable contributions leading to interference. This is supported by an increased contrast of about 0.5 [Fig. 2(b)] and a different $\phi$ dependence; see Figs. 1 and 2(c).

In Fig. 2(c) the low-energy part of the delay scan is compared with the predictions for the positions of maximum signal made by classical and QO models with $f(t)=1$. They are obtained by first finding the ionization instants $t_{\mathrm{i}}$ for every final momentum $p_{x}$. Here, we neglect the second harmonic field, using only the fundamental field in

$$
\frac{\left(p_{x}+A_{x}\left(t_{\mathrm{i}}\right)\right)^{2}}{2}+\varepsilon_{0}=0,
$$

where $\varepsilon_{0}=0$ for the classical model and $\varepsilon_{0}=I_{\mathrm{p}}$ in the QO case. Ionization is strongest for zero transverse initial velocity, i.e., $\operatorname{Re}\left[v_{y, \mathrm{i}}\right]=\operatorname{Re}\left[p_{y}+A_{y}\left(t_{\mathrm{i}}, \phi\right)\right]=0$, where the real part is used because $t_{\mathrm{i}}$ is complex in the QO model [21]. Therefore, we find the delay $\phi_{\mathrm{i}}\left(p_{x}\right)$ maximizing the signal on axis $\left(p_{y}=0\right)$ by solving

$$
\operatorname{Re}\left[A_{y}\left(t_{\mathrm{i}}, \phi_{\mathrm{i}}\right)\right]=0 .
$$

In both models, two branches can be seen, corresponding to ionization times $t_{\mathrm{i}, a}$ in the ascending and $t_{\mathrm{i}, b}$ in the descending part of the fundamental electric field. These contributions are known as short and long ATI trajectories [37]. Electrons in the ascending branch (blue [gray] solid line crossing $p_{x}=0$ at $\phi=0$ ) are born during an ascending electric field (short trajectories). These electrons cannot recollide with the ion [3]. The results from the TDSE suggest a major contribution from the descending branch, corresponding to trajectories starting in an descending field, which can revisit the core (long trajectories). Their dominance might be caused by Coulomb focusing [38], as in holography [4]. The rapid oscillations, however, hint at an interference of both branches.

\section{B. Intracycle interference}

To explain the rapid oscillations for the slow electrons in Fig. 2(c) we find the delay values at the TDSE delay-scan maxima. We compare them with the momentum spectrum along the $x$ axis from ionization with the 800-nm single-color field (integrated over $p_{y}$ ); see Figs. 3(a) and 3(b). It is striking that peaks in the spectrum are in good coincidence with extrema in the oscillations of the delay value. These peaks can be explained by a constructive intracycle interference of the weak signal from the short ATI trajectories with the dominant long ATI trajectories. We take these two contributions as complex amplitudes $a(\phi)$ and $b(\phi)$ corresponding to the ascending and descending branch, respectively. A measured signal shows the interference $|a+b|^{2}=|a|^{2}+$ $|b|^{2}+2|a||b| \cos \left(\varphi_{a}-\varphi_{b}\right)$, with phases $\varphi_{a}$ and $\varphi_{b}$. We assume $|a|$ to be small enough to neglect $|a|^{2}$, whereas the interference is linear in $|a|$ and thus not necessarily negligible. A peak in the electron spectrum indicates constructive interference $|b|^{2}+2|a||b|$. In the two-color scheme this corresponds to a shift of the maximum to smaller $\phi$, if the maximum of $|a(\phi)|$ lies at smaller $\phi$ but within a range of less than $\pi / 2$ from the maximum of $|b(\phi)|$. A minimum in the spectrum $|b|^{2}-2|a||b|$ accordingly leads to a shift of the maximum

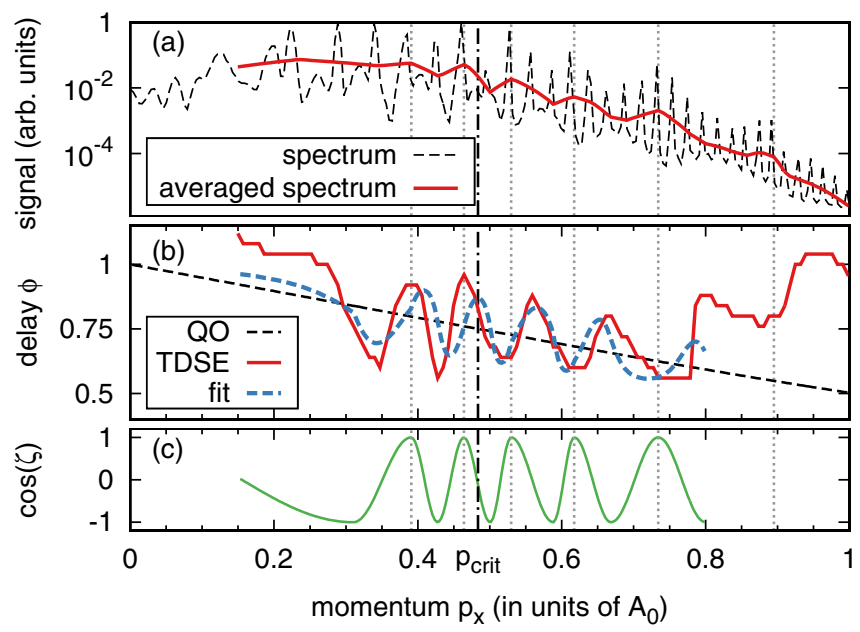

FIG. 3. (Color online) Comparison of the spectrum from the 800 -nm single-color field and observables from the two-color calculation. (a) Single-color spectrum before (black dashed line) and after averaging over the ATI peaks (red [gray] solid line). (b) Positions of the maxima in Fig. 2 (red [gray] solid line), compared to the maximum of $|b|$ (black dashed line) and a modified QO model (blue [gray] dashed line). (c) Interference term extracted from the averaged TDSE spectrum in panel (a) with the maxima highlighted by gray dotted vertical lines.

in opposite direction. From the QO model in Fig. 2(c) this behavior is expected for $p_{x}>p_{\text {crit }} \approx 0.48 A_{0}$, while below $p_{\text {crit }}$ the shift directions are reversed (see also Fig. 3).

To quantify the explained effects we calculate the semiclassical action [19]

$$
S(\mathbf{p}, \phi)=-\frac{1}{2} \int_{t_{\mathrm{i}}}\left[\mathbf{p}+\mathbf{A}\left(t^{\prime}, \phi\right)\right]^{2} d t^{\prime}+I_{\mathrm{p}} t_{\mathrm{i}},
$$

and insert the complex ionization times obtained from Eq. (3) for the QO model to get $a=\exp \left(i S_{a}\right)$ and $b=\exp \left(i S_{b}\right)$. The maximum of $|a|(|b|)$ along $\phi$ now corresponds exactly to the ascending (descending) line in Fig. 2. Calculation of the intracycle interference with this model [35] does not reproduce the TDSE result. This could be corrected by including Coulomb effects in the SFA [37,39]. Instead, we extract the phase difference $\zeta\left(p_{x}\right)$ between the two branches from the TDSE single-color ATI result in Fig. 3(a), which intrinsically contains Coulomb effects. To this end, we find the local extrema after dividing by the rapidly decreasing smoothed signal (averaged over intracycle structures). Moving from zero to high momentum we assign each maximum $n$ at position $p_{x}(n)$ (gray dotted vertical lines) a value of $\zeta\left(p_{x}(n)\right)=2 \pi(n-1)$ and each minimum $m$ the value $\zeta\left(p_{x}(m)\right)=\pi[2(m-1)+1]$. Intermediate values are linearly interpolated and the cosine of the resulting phase difference is displayed in Fig. 3(c). We then write the complex amplitudes as

$$
\begin{gathered}
a^{\prime}=|a| \exp \left\{-i \frac{1}{2} \operatorname{Re}\left[\int_{t_{\mathrm{i}, a}} A_{y}\left(t^{\prime}, \phi\right)^{2} d t^{\prime}\right]\right\}, \\
b^{\prime}=|b| \exp \left\{i \zeta\left(p_{x}\right)-i \frac{1}{2} \operatorname{Re}\left[\int_{t_{\mathrm{i}, b}} A_{y}\left(t^{\prime}, \phi\right)^{2} d t^{\prime}\right]\right\} .
\end{gathered}
$$


This includes the phase caused by the second-color field to retain the delay dependence in the phase.

We perform a least-square fit of the model signal $\left|M\left(p_{x}, \phi\right)\right|^{2}=\left|a^{\prime}+\beta b^{\prime}\right|^{2}$ to the two-color TDSE from Fig. 2(c). The single parameter $\beta$ quantifies the relative strength of the two branches. The delay values at the maxima of the obtained delay dependence are depicted in Fig. 3(b). We restrict the fit to momenta $p_{x}<0.8 A_{0}$ as the model does not cover the above-mentioned interference with fast electrons. The fit parameter is returned as $\beta \approx 2.3$.

It is also possible to estimate $\beta$ from the modulation depth of the intracycle interference in the electron spectrum of the 800-nm field. After dividing by the smoothed signal as above, we use the ratio of subsequent maxima and minima to obtain $\beta$ at the corresponding momentum values. In the momentum range $0.4 A_{0}<p_{x}<0.8 A_{0}$ we find values of $\beta \approx 5$. These higher values might originate from a background due to other ionization pathways, which affects the modulation depth. Thus, the two-color-based retrieval has two advantages: (i) it is unaffected by such a background, as we scale both the TDSE and the model distribution to the range between zero and one and (ii) it allows us to directly identify the long-trajectory branch as the stronger contribution. Similar results are obtained for the two calculations with higher intensity and with krypton.

\section{Extraction of ionization times}

Importantly, we can use the two-color TDSE results for $p_{y}=0$ to extract the ionization times. Inverting Eq. (4) yields

$$
\operatorname{Re}\left[t_{\mathrm{i}}\left(p_{x}\right)\right]=\frac{1}{2 \omega}\left[n \pi-\phi_{\mathrm{i}}\left(p_{x}\right)\right] .
$$

For this particular choice of observation no knowledge about laser intensity $E_{0}$ or ionization potential $I_{\mathrm{p}}$ is necessary to obtain $\operatorname{Re}\left[t_{\mathrm{i}}\right]$. Also, the result is independent of $\operatorname{Im}\left[t_{\mathrm{i}}\right]$, unlike the situation in HHG [21]. The integer $n$ has to be chosen appropriately to match the correct laser quarter cycle (here $n=1$ ). Our understanding of the oscillations allows us to extract the dominant branch from the TDSE scan. By applying a low-pass filter in the Fourier space conjugated to $p_{x}$ to the maximum positions $\phi_{\mathrm{i}}\left(p_{x}\right)$ we obtain a smoothed curve shown in Fig. 4(b). Inserting this into Eq. (8) then yields the ionization times shown in Fig. 4(a), which in the momentum range $0.4 A_{0}<p_{x}<0.75 A_{0}$ follows closely the predictions made by the QO model. The mean deviation from the QO model in this range is about 11 as. Thus, measurement of the electron momentum distribution in the presented two-color scheme allows the extraction of ionization times for those electrons that revisit the ion core. These trajectories begin up to 110 as earlier than predicted by the classical model. If conditions can be found where the short trajectories can be identified in the delay scan, our scheme will allow us to measure their ionization times, which are inaccessible by the two-color HHG schemes used in Refs. [18,21].

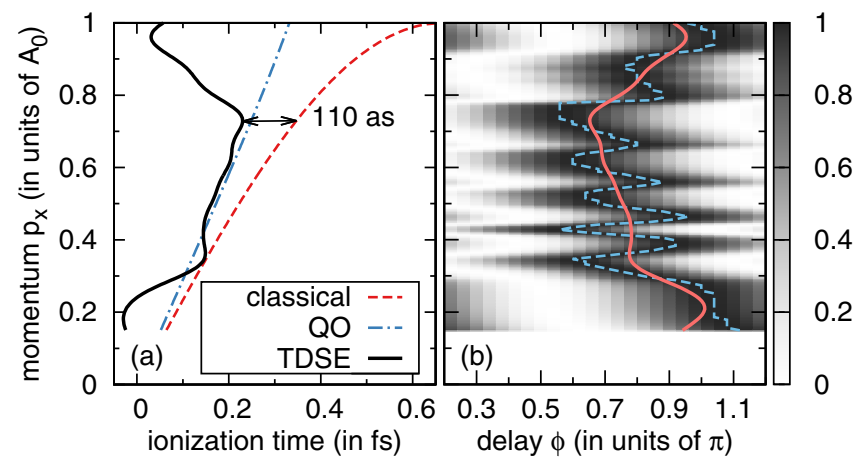

FIG. 4. (Color online) (a) Comparison of reconstructed and predicted ionization times. (b) Delay scan from Fig. 2 for the slow electrons, with the original (blue [gray] dashed line) and smoothed (red [gray] solid line) maxima $\phi_{\mathrm{i}}\left(p_{x}\right)$.

For the fast electrons we use a classical model where we solve the equation of motion for $x$ to find the first recollision time $t_{\mathrm{r}}$ from the condition $x\left(t_{\mathrm{i}}, t_{\mathrm{r}}\right)=0$ for every ionization time $t_{\mathrm{i}}$. Inserting these times into the condition $y\left(t_{\mathrm{i}}, t_{\mathrm{r}}, \phi_{\mathrm{r}}\right)=0$ gives the delay $\phi_{\mathrm{r}}$ that allows recollision in both dimensions. Assuming that the electron can be scattered into any direction [40], we select the trajectory with $p_{y}=0$ and find the final momentum $p_{x}$; see the curve in the high-energy region of Fig. 2(c). In the figure, we distinguish into short and long trajectories as in HHG; i.e., the long trajectories have a decreasing return energy over return time. We find a good agreement of the TDSE delay scan with the long trajectories in the model. While HHG experiments normally focus on the short trajectories, we explain the dominance of the long trajectories $[41,42]$ in the present case by the higher ionization rate and the absence of phase matching.

\section{SUMMARY}

In summary, we have investigated the ionization process in a two-color field with perpendicular polarizations by solving the time-dependent Schrödinger equation. Selecting electrons with zero lateral final momentum reveals rapid oscillations for the slow electrons. We identify the main signal as the recolliding electrons, but the recollison-free short-trajectory electrons become visible through oscillations due to intracycle interference. From these oscillations we find the relative strengths of the contributing trajectories. In addition, the two-color scheme gives access to the ionization times for the electrons with fewer assumptions than in the HHG experiments. It is therefore a powerful tool to investigate electron dynamics on the attosecond time scale.

\section{ACKNOWLEDGMENTS}

We thank the Deutsche Forschungsgemeinschaft for financial support.
[1] P. B. Corkum, N. H. Burnett, and F. Brunel, Phys. Rev. Lett. 62, 1259 (1989).
[2] J. L. Krause, K. J. Schafer, and K. C. Kulander, Phys. Rev. Lett. 68, 3535 (1992). 
[3] P. B. Corkum, Phys. Rev. Lett. 71, 1994 (1993).

[4] Y. Huismans, A. Rouzée, A. Gijsbertsen, J. H. Jungmann, A. S. Smolkowska, P. S. W. M. Logman, F. Lépine, C. Cauchy, S. Zamith, T. Marchenko, J. M. Bakker, G. Berden, B. Redlich, A. F. G. V. D. Meer, H. G. Muller, W. Vermin, K. J. Schafer, M. Spanner, M. Y. Ivanov, O. Smirnova, D. Bauer, S. V. Popruzhenko, and M. J. J. Vrakking, Science 331, 61 (2011).

[5] X. B. Bian, Y. Huismans, O. Smirnova, K. J. Yuan, M. J. J. Vrakking, and A. D. Bandrauk, Phys. Rev. A 84, 043420 (2011).

[6] X. Xie, K. Doblhoff-Dier, S. Roither, M. S. Schöffler, D. Kartashov, H. Xu, T. Rathje, G. G. Paulus, A. Baltuska, S. Gräfe, and M. Kitzler, Phys. Rev. Lett. 109, 243001 (2012).

[7] N. Dudovich, O. Smirnova, J. Levesque, Y. Mairesse, M. Y. Ivanov, D. M. Villeneuve, and P. B. Corkum, Nat. Phys. 2, 781 (2006).

[8] J. Itatani, J. Levesque, D. Zeidler, H. Niikura, H. Pépin, J. C. Kieffer, P. B. Corkum, and D. M. Villeneuve, Nature (London) 432, 867 (2004).

[9] D. Shafir, Y. Mairesse, D. M. Villeneuve, P. B. Corkum, and N. Dudovich, Nat. Phys. 5, 412 (2009).

[10] S. Baker, J. S. Robinson, C. A. Haworth, H. Teng, R. A. Smith, C. C. Chirilă, M. Lein, J. W. G. Tisch, and J. P. Marangos, Science 312, 424 (2006).

[11] O. Smirnova, Y. Mairesse, S. Patchkovskii, N. Dudovich, D. Villeneuve, P. Corkum, and M. Y. Ivanov, Nature (London) 460, 972 (2009).

[12] S. Haessler, J. Caillat, W. Boutu, C. Giovanetti-Teixeira, T. Ruchon, T. Auguste, Z. Diveki, P. Breger, A. Maquet, B. Carré, R. Taïeb, and P. Salières, Nat. Phys. 6, 200 (2010).

[13] T. Weber, H. Giessen, M. Weckenbrock, G. Urbasch, A. Staudte, L. Spielberger, O. Jagutzki, V. Mergel, M. Vollmer, and R. Dörner, Nature (London) 405, 658 (2000).

[14] R. Moshammer, B. Feuerstein, W. Schmitt, A. Dorn, C. D. Schröter, J. Ullrich, H. Rottke, C. Trump, M. Wittmann, G. Korn, K. Hoffmann, and W. Sandner, Phys. Rev. Lett. 84, 447 (2000).

[15] I. J. Kim, C. M. Kim, H. T. Kim, G. H. Lee, Y. S. Lee, J. Y. Park, D. J. Cho, and C. H. Nam, Phys. Rev. Lett. 94, 243901 (2005).

[16] M. Kitzler and M. Lezius, Phys. Rev. Lett. 95, 253001 (2005).

[17] L. Zhang, X. Xie, S. Roither, Y. Zhou, P. Lu, D. Kartashov, M. Schöffler, D. Shafir, P. B. Corkum, A. Baltuska, A. Staudte, and M. Kitzler, Phys. Rev. Lett. 112, 193002 (2014).

[18] D. Shafir, H. Soifer, B. D. Bruner, M. Dagan, Y. Mairesse, S. Patchkovskii, M. Y. Ivanov, O. Smirnova, and N. Dudovich, Nature (London) 485, 343 (2012).

[19] M. Lewenstein, P. Balcou, M. Y. Ivanov, A. L'Huillier, and P. B. Corkum, Phys. Rev. A 49, 2117 (1994).

[20] P. Salières, B. Carré, L. L. Déroff, F. Grasbon, G. G. Paulus, H. Walther, R. Kopold, W. Becker, D. B. Milosević, A. Sanpera, and M. Lewenstein, Science 292, 902 (2001).

[21] J. Zhao and M. Lein, Phys. Rev. Lett. 111, 043901 (2013).
[22] M. Murakami, O. Korobkin, and M. Horbatsch, Phys. Rev. A 88, 063419 (2013).

[23] L. Zhang, X. Xie, S. Roither, D. Kartashov, Y. L. Wang, C. L. Wang, M. Schöffler, D. Shafir, P. B. Corkum, A. Baltuska, I. Ivanov, A. Kheifets, X. J. Liu, A. Staudte, and M. Kitzler, Phys. Rev. A 90, 061401 (2014).

[24] M. Richter, M. Kunitski, M. Schöffler, T. Jahnke, L. P. H. Schmidt, M. Li, Y. Liu, and R. Dörner, Phys. Rev. Lett. 114, 143001 (2015).

[25] D. Ray, Z. Chen, S. De, W. Cao, I. V. Litvinyuk, A. T. Le, C. D. Lin, M. F. Kling, and C. L. Cocke, Phys. Rev. A 83, 013410 (2011).

[26] X. Xie, S. Roither, D. Kartashov, E. Persson, D. G. Arbó, L. Zhang, S. Gräfe, M. S. Schöffler, J. Burgdörfer, A. Baltuska, and M. Kitzler, Phys. Rev. Lett. 108, 193004 (2012).

[27] X. Xie, S. Roither, S. Gräfe, D. Kartashov, E. Persson, C. Lemell, L. Zhang, M. S. Schöffler, A. Baltuska, J. Burgdörfer, and M. Kitzler, New J. Phys. 15, 043050 (2013).

[28] D. G. Arbó, S. Nagele, X. M. Tong, X. Xie, M. Kitzler, and J. Burgdörfer, Phys. Rev. A 89, 043414 (2014).

[29] D. G. Arbó, J. Phys. B 47, 204008 (2014).

[30] J. Chen, S. Zhao, G. Wang, and X. Zhou, J. Phys. B 47, 245601 (2014).

[31] J. Liu, W. Chen, B. Zhang, J. Zhao, J. Wu, J. Yuan, and Z. Zhao, Phys. Rev. A 90, 063420 (2014).

[32] S. Skruszewicz, J. Tiggesbäumker, K. Meiwes-Broer, M. Arbeiter, T. Fennel, and D. Bauer, arXiv:1502.00614 [physics:quant-ph].

[33] W. Becker, F. Grasbon, R. Kopold, D. B. Milosević, G. G. Paulus, and H. Walther, in Advances in Atomic, Molecular, and Optical Physics, Vol. 48, edited by B. Bederson and H. Walther (Academic Press, San Diego, CA, 2002), pp. 35-98.

[34] F. Lindner, M. G. Schätzel, H. Walther, A. Baltuska, E. Goulielmakis, F. Krausz, D. B. Milosević, D. Bauer, W. Becker, and G. G. Paulus, Phys. Rev. Lett. 95, 040401 (2005).

[35] D. G. Arbó, K. L. Ishikawa, K. Schiessl, E. Persson, and J. Burgdörfer, Phys. Rev. A 81, 021403 (2010).

[36] J. Henkel, M. Lein, and V. Engel, Phys. Rev. A 83, 051401 (2011)

[37] T. M. Yan and D. Bauer, Phys. Rev. A 86, 053403 (2012).

[38] T. Brabec, M. Y. Ivanov, and P. B. Corkum, Phys. Rev. A 54, R2551 (1996).

[39] S. Popruzhenko and D. Bauer, J. Mod. Opt. 55, 2573 (2008).

[40] G. G. Paulus, W. Becker, W. Nicklich, and H. Walther, J. Phys. B 27, L703 (1994).

[41] M. Meckel, D. Comtois, D. Zeidler, A. Staudte, D. Pavicić, H. C. Bandulet, H. Pépin, J. C. Kieffer, R. Dörner, D. M. Villeneuve, and P. B. Corkum, Science 320, 1478 (2008).

[42] L. Brugnera, D. J. Hoffmann, T. Siegel, F. Frank, A. Zaïr, J. W. G. Tisch, and J. P. Marangos, Phys. Rev. Lett. 107, 153902 (2011). 